\title{
An X-ray Emitting Black Hole in a Globular Cluster
}

\author{
T.J. Maccarone ${ }^{1}$, G. Bergond ${ }^{2}$, A. Kundu ${ }^{3}$, K.L. Rhode ${ }^{4,5,6}$, J.J. \\ Salzer $^{4,5}$, I.C. Shih ${ }^{3}$ and S.E. Zepf ${ }^{3}$ \\ ${ }^{1}$ School of Physics and Astronomy, University of Southampton, Southampton, UK, SO16 4ES \\ email: tjm@phys.soton.ac.uk \\ ${ }^{2}$ Instituto de Astrofísica de Andalucía (IAA/CSIC), Camino Bajo de Hué tor 50, 18008 \\ Granada, Spain \\ ${ }^{3}$ Department of Physics and Astronomy, Michigan State University, East Lansing, MI 48824, \\ USA \\ ${ }^{4}$ Department of Astronomy, Indiana University, Bloomington, IN 47405, USA \\ ${ }^{5}$ Department of Astronomy, Wesleyan University, Middletown, CT, 06459, USA \\ ${ }^{6}$ Department of Astronomy, Yale University, New Haven CT, 06520, USA
}

\begin{abstract}
We present optical and X-ray data for the first object showing strong evidence for being a black hole in a globular cluster. We show the initial X-ray light curve and X-ray spectrum which led to the discovery that this is an extremely bright, highly variable source, and thus must be a black hole. We present the optical spectrum which unambiguously identifies the optical counterpart as a globular cluster, and which shows a strong, broad [O III] emission line, most likely coming from an outflow driven by the accreting source.
\end{abstract}

Keywords. X-ray: binaries, globular clusters: general, galaxies: individual (NGC 4472)

\section{Introduction}

Since the early days of X-ray astronomy, there has been considerable debate over whether globular clusters contained black holes. With the discovery of Type I X-ray bursts from all globular clusters in the Milky Way with bright X-ray sources (starting with Grindlay et al. 1976), and their subsequent explanation as episodes of thermonuclear burning on the surfaces of neutron stars (Woosley \& Taam 1976; Swank et al. 1977), it became clear that there was no evidence for any accreting black holes in the Milky Way's globular cluster system.

Interpretations of the observations have been taken in two directions. One is simply that given only 13 bright X-ray sources in the Milky Way's globular cluster system, it is not so unlikely for them all to have neutron star accretors, especially in light of the fact that about 10 times as many neutron stars as black holes are expected to be produced for most stellar initial mass functions. The alternative is that dynamical effects are responsible for ejecting black holes from globular clusters. Severe mass segregation is likely to take place for globular cluster black holes, as they should be many times heavier than all the other stars in the cluster. This can lead to the formation of a "cluster within a cluster" where the heaviest stars (i.e. the black holes) feel negligible effects from the other stars in the cluster, which in turn leads to a cluster with a short evaporation timescale (Spitzer 1969). Numerical calculations have found that this evaporation can be accelerated further due to binary processes (e.g Portegies Zwart \& McMillan 2000).

Early results from the Chandra X-ray Observatory gave new hope that globular cluster black holes might be detectable, by opening up the window of looking in other galaxies. 
Previously, only ROSAT could resolve point sources in other galaxies, and its localization of sources was generally not good enough to allow for unique identification of optical counterparts. The first few years of Chandra observations revealed several extragalactic globular cluster X-ray sources brighter than the Eddington limit for a neutron star (e.g. Angelini et al. 2001; Kundu et al. 2002), but a globular cluster may contain multiple bright neutron stars (as does, for example M 15 in our own galaxy - White \& Angelini 2001), and that the quality of X-ray spectra available from Chandra for even the brightest extragalactic sources is insufficient to make phenomenological determinations that a source has a black hole accretor. It was pointed out that only large amplitude variability could prove that we were seeing the emission from a single source, rather than multiple sources (Kalogera, King \& Rasio 2003).

Furthermore, the optical catalogs used to identify globular cluster counterparts to Xray sources have been predominantly photometric catalogs, sometimes made even without color selections being used to ensure that that the optical source in question truly is a globular cluster. Most studies done to date have focused on HST images of the central regions of elliptical galaxies with high specific frequencies of globular clusters. In these regions, and with the angular resolution of HST, contamination will be rare, especially if color cuts are used to ensure that the contribution of background quasars is minimized. In the halos of galaxies, the surface density of real globular clusters will drop, and contamination will be a more serious problem. In either case, when one is looking for conclusive proof that an object is a globular cluster black hole, spectroscopic confirmation that the object is a globular cluster is essential - the fractional contamination of the X-ray sources due to background AGN will be more serious at very high fluxes, corresponding to luminosities above $10^{39} \mathrm{ergs} / \mathrm{sec}$ than it will at lower levels consistent bright neutron star accretors. Furthermore, the optical to X-ray ratios for globular cluster black holes near the Eddington limit and background quasars are quite similar.

\section{Discovery of a globular cluster black hole}

In a recent paper, we found a source meeting all the strict criteria for identifying an X-ray emitting black hole in a globular cluster (Maccarone et al. 2007). XMMU $\mathrm{J} 1229397+075333$ has an X-ray luminosity of $4.5 \times 10^{39} \mathrm{ergs} / \mathrm{sec}$. It shows variability of a factor of 7 in count rate, in a time span of about 3 hours (see Fig. 1). It is located in a spectroscopically selected globular cluster. The object was first detected by ROSAT, and is included in the intermediate luminosity X-ray source catalog of Colbert \& Ptak (2002), but as it is about 7 arcminutes from the center of NGC 4472, its host galaxy, good optical follow-up, combined with the positional accuracy obtained from archival Chandra data were necessary to ensure that it was not just a background active galactic nucleus.

\section{Observational properties}

This source has several unusual X-ray properties, all of which give some clues about its possible nature. Its X-ray spectrum is dominated by a very soft, quasi-thermal component. The best fitting models give inner disk radii of thousands of kilometers, with temperatures of about $0.2 \mathrm{keV}$. The change in X-ray count rate is predominately a change in the low energy X-ray emission, and is consistent with a change only in foreground absorption. This source was also observed in 1994 by ROSAT, and in 2000 by Chandra, in both cases as part of observations of the whole of NGC 4472. In both of these cases, the X-ray luminosity was within a factor of a few of $4 \times 10^{39} \mathrm{ergs} / \mathrm{sec}$, with bigger uncertainty 


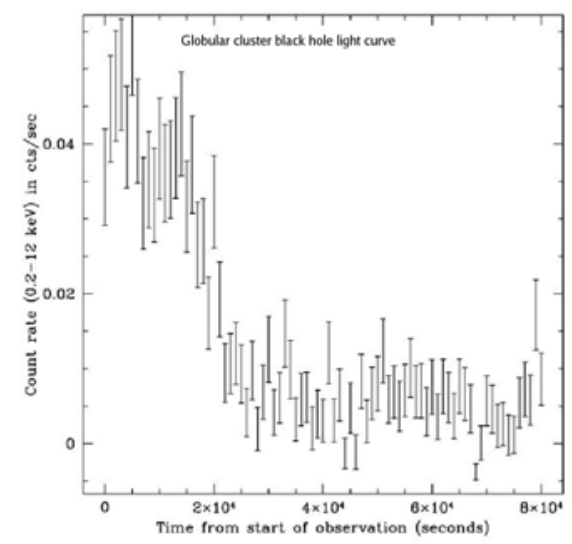

Figure 1. The XMM-Newton light curve of XMMU J1229397+07533.

in the luminosities than for XMM due to the lower count rates, narrower spectral energy range, and, in the case of ROSAT, much poorer spectral resolution, when compared with XMM. This suggests strongly that this source has been persistently bright for at least a 12 year period. The comparison of the different epochs' spectra and detailed spectral fitting can be found in Shih et al. (2007).

The optical spectrum also shows an unusual feature which may shed light on the nature of the accretor. Two optical spectra have been taken of this object, and in each case, a strong, broad [O III] emission line is seen (see Fig. 2 for one example). Unfortunately, the observations were aimed at making spectroscopic confirmation of the globular cluster nature of a large number of clusters, and at doing kinematic studies of the clusters. This means that the spectra have been taken with relatively high spectral resolution, but over a relatively narrow wavelength range, and this wavelength range includes only [O III] 5007 $\AA$ among the emission lines which are commonly strong. Emission lines are rarely seen in globular clusters' integrated spectra, and are normally attributed to planetary nebulae when they are seen. The line is many times broader in velocity profile than lines from planetary nebulae. A detailed description of the line properties can be found in Zepf et al. (2007).

\section{Interpretation: the nature of this object}

The large inferred inner disk radius from the X-ray spectrum of this object implies that one of two things is most likely to be true. Either the object contains an intermediate mass black hole of a few hundred solar masses accreting at about a few percent of its Eddington rate, or it contains a stellar mass black hole accreting at a mildly super-Eddington rate. In the former case, the combination of observed temperature and luminosity is straightforward, as it requires only that the accretion disk extend in to the innermost stable circular orbit for the black hole.

The latter case, while not as intuitively obvious, is equally plausible physically. In the event of super-Eddington accretion, one can expect annuli where the luminosity is the local Eddington luminosity. This yields a total luminosity of the Eddington luminosity multiplied by the logarithm of the ratio of the mass accretion rate to the accretion rate needed to reach the Eddington luminosity (see e.g. Begelman, King \& Pringle 2006). While the spectrum will not any more be exactly that predicted by the standard disk blackbody model, with moderate quality data like those which exist for extragalactic 

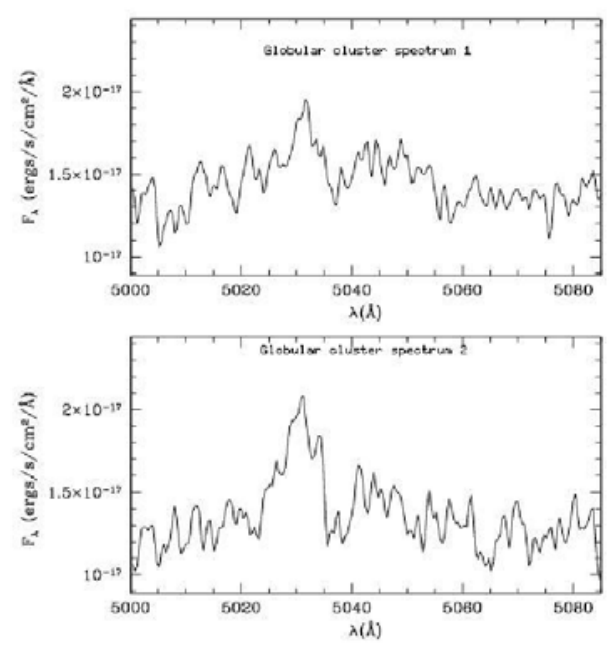

Figure 2. The optical spectrum of RZ2109, the globular cluster containing XMMU J1229397+07533. The emission line appears slightly redshifted from the position of [O III] at $5007 \AA$, with the redshift the same for the line as it is for the absorption lines in the stellar continuum. The two different epochs of VLT spectra are shown.

X-ray binaries, this model will provide an acceptable fit, and will yield an inner disk radius equal to the radius of the outermost annulus where the source is locally Eddington limited. Taking this into account, our data are well fit by a model in which the black hole mass is about $10 M_{\odot}$, and the accretion rate is about 40 times what would be needed to produce an Eddington-luminosity source (see e.g. Begelman, King \& Pringle 2006).

The variability can be explained by a puffed up warped region in the accretion disk which precesses, sometimes obscuring the central region of the accretion flow, while, most of the time, it is not blocking our line of sight to the source (see Shih et al. 2007 for a more detailed discussion of the source's X-ray variability). A precession period of about 100 days, combined with a radius of about $10^{11} \mathrm{~cm}$ for the location of the warp, gives a good match to the timescale of about 3 hours on which the obscuration takes place. One could alternatively consider self-obscuration by a variable disk wind, but it seems unlikely that the observed column density would change from a level which is negligible compared to the foreground column density of $1.6 \times 10^{20} \mathrm{~cm}^{-2}$ to the level of approximately $3 \times 10^{21}$ $\mathrm{cm}^{-2}$ needed to match the spectrum in the faint part of the light curve. Strongly warped accretion disks which are puffed up enough to allow self-obscuration are most likely to be seen for luminosities near the Eddington luminosity, so the interpretation of the source as a super-Eddington stellar mass black hole is favored in this scenario. Given the long inferred precession timescale, despite the high mass accretion rate which speeds the transfer of warp angular momentum, we expect such a system to have a relatively long orbital period of $\sim 1$ month. This is in good agreement with the finding that the object has been a bright X-ray source for over a decade, as long period systems show long outbursts followed by extremely long periods of quiescence (e.g. Portegies Zwart, Dewi \& Maccarone 2005 and references within).

The optical spectrum is consistent with the idea that this system contains a superEddington stellar mass black hole, rather than an intermediate mass black hole (see Zepf et al. 2007 for a more detailed discussion of the cluster's optical spectrum). The broad [O III] emission line can be explained as the result of a bubble being inflated by strong 
disk winds from a stellar mass black hole exceeding the Eddington limit. This bubble then collides with the interstellar medium in the globular cluster, producing a shock. The shock velocity will approximately equal the physical width of the emission line. The shock velocity can be slowed down from the original outflow velocity to the observed velocity in a reasonable duration, given expected density of the interstellar medium in a globular cluster. Other scenarios for producing a broad line, especially from an intermediate mass black hole, run into problems - while [O III] can come from photoionization, and the line width could be due to virial motions near the black hole, it is difficult (albeit not impossible) to allow for enough material to be located close enough to the black hole to match both the line luminosity and the line width. Thus, an outflow would still be required in the case of an intermediate mass black hole, and outflows are generally quite weak for sources with luminosities well below their Eddington limits (Proga 2007).

\section{Conclusions}

We have shown for the first time clear evidence of a globular cluster black hole, on the basis of strong, highly variable X-ray emission from a source in a spectroscopically confirmed globular cluster. Based on the X-ray spectrum, the characteristic variability, and the [O III] emission in the optical spectrum, the black hole is most likely a stellar mass object accreting far faster than its Eddington rate. Given that it is difficult to develop a scenario in which a globular cluster could have both a stellar mass black hole in a binary and an intermediate mass black hole, this argues against the idea that all globular clusters contain intermediate mass black holes. This discovery also motivates future searches for quiescent stellar mass black holes in the Milky Way's globular clusters; these may be hiding among the X-ray sources currently classified as cataclysmic variable stars. Radio emission should be detectable only from quiescent stellar mass black holes, and should be the one feasible discriminant between the two classes of systems.

\section{Acknowledgements}

We are grateful to Tom Dwelly, Sebastian Jester, Elmar Körding, Phil Charles, Mike Eracleous, Steinn Sigurdsson, Robin Barnard, Andrew King, Guillaume Dubus and Mark Voit for useful discussions.

\section{References}

Angelini, L., Loewenstein, M., Mushotzky, R. F., 2001, ApJL, 557L, 35

Begelman, M. C., King, A. R., Pringle, J. E., 2006, MNRAS, 370, 399

Colbert, E. J. M. \& Ptak, A. F., 2002, ApJS 143, 25

Grindlay, J., Gursky, H., Schnopper, H., Parsignault, D. R., Heise, J., Brinkman, A. C., \& Schrijver, J. 1976, ApJL, 205, L127

Kalogera, V., King, A. R., Rasio, F. A., 2004, ApJL, 600, L17

Kundu, A., Maccarone, T. J., Zepf, S. E., 2002, ApJL, 574, L5

Maccarone, T. J., Kundu, A., Zepf, S. E., \& Rhode, K. L. 2007, Nature, 445, 183

Portegies Zwart, S. F., Dewi, J., \& Maccarone, T. 2004, MNRAS, 355, 413

Portegies Zwart, S. F., \& McMillan, S. L. W. 2000, ApJL, 528, L17

Proga, D. 2007, The Central Engine of Active Galactic Nuclei in press

Spitzer, L. J. 1969, ApJL, 158, L139

Swank, J. H., Becker, R. H., Boldt, E. A., Holt, S. S., Pravdo, S. H., \& Serlemitsos, P. J. 1977, ApJL, 212, L73

White, N. E. \& Angelini, L., 2001 ApJL, 561, 101L

Woosley, S. E. \& Taam, R. E. 1976, Nature, 263, 101 